\title{
Time from first seen in specialist care to surgery does not influence survival outcome in patients with upfront resected pancreatic adenocarcinoma
}

\author{
M. Brugel ${ }^{1 *}$, O. Bouché ${ }^{1,2}$, R. Kianmanesh ${ }^{2,3}$, L. Teuma ${ }^{3}$, A. Tashkandi ${ }^{3}$, J. M. Regimbeau ${ }^{4,5}$, P. Pessaux ${ }^{6,7}$, B. Royer ${ }^{8}$, \\ R. Rhaiem ${ }^{3}$, C. Perrenot ${ }^{2,3}$, C. Neuzillet ${ }^{9,10}$, T. Piardi $^{2,3}$ and S. Deguelte ${ }^{2,3}$
}

\begin{abstract}
Background: This study evaluated the impact of time to surgery (TTS) on overall survival (OS), disease free survival (DFS) and postoperative complication rate in patients with upfront resected pancreatic adenocarcinoma (PA).

Methods: We retrospectively included patients who underwent upfront surgery for PA between January 1, 2004 and December 31, 2014 from four French centers. TTS was defined as the number of days between the date of the first consultation in specialist care and the date of surgery. DFS for a 14-day TTS was the primary endpoint. We also analyzed survival depending on different delay cut-offs (7, 14, 28, 60 and 75 days).

Results: A total of 168 patients were included. 59 patients (35\%) underwent an upfront surgery within 14 days. Patients in the higher delay group (> 14 days) had significantly more vein resections and endoscopic biliary drainage. Adjusted OS $(p=0.44)$, DFS $(p=0.99)$, fistulas $(p=0.41)$, hemorrhage $(p=0.59)$ and severe post-operative complications ( $p=0.82$ ) were not different according to TTS ( $>14$ days). Other delay cut-offs had no impact on OS or DFS.
\end{abstract}

Discussion: TTS seems to have no impact on OS, DFS and 90-day postoperative morbidity.

Keywords: Pancreatic adenocarcinoma, Resectable, Delay, Time to surgery

\section{Introduction}

Pancreatic adenocarcinoma (PA) is one of the most aggressive digestive cancers. Five-year overall survival (OS) rate is estimated below $8 \%$ (all stages combined) [1]. This malignancy is expected to be the second leading cause of cancer-related death in Europe by 2030[2].

French actual standard of care for resectable PA is upfront carcinologic surgery followed by adjuvant chemotherapy [3]. Unfortunately, prognosis remains poor

\footnotetext{
*Correspondence: mbrugel@chu-reims.fr

1 Department of Ambulatory Oncology Care Unit, Centre Hospitalier

Universitaire de Reims, Rue du general Koenig, Reims, France

Full list of author information is available at the end of the article
}

despite improvements in surgical technique, perioperative care, diagnosis accuracy, patient selection and more active chemotherapy regimen [4]. New perspectives are needed to increase both survival rates and quality of life for patients diagnosed with PA.

One of the objectives studied in other cancers has been to reduce time to treatment by improving the organization of the care pathway. Time to surgery (TTS) has turned out to be a major prognostic factor associated with survival in several malignancies [5-8]. To date, the impact of TTS on OS and disease-free survival (DFS) in patients diagnosed with resectable PA remains unclear [9].

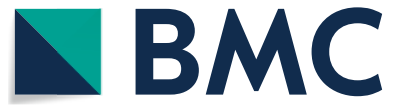

(c) The Author(s) 2021. Open Access This article is licensed under a Creative Commons Attribution 4.0 International License, which permits use, sharing, adaptation, distribution and reproduction in any medium or format, as long as you give appropriate credit to the original author(s) and the source, provide a link to the Creative Commons licence, and indicate if changes were made. The images or other third party material in this article are included in the article's Creative Commons licence, unless indicated otherwise in a credit line to the material. If material is not included in the article's Creative Commons licence and your intended use is not permitted by statutory regulation or exceeds the permitted use, you will need to obtain permission directly from the copyright holder. To view a copy of this licence, visit http://creativecommons.org/licenses/by/4.0/. The Creative Commons Public Domain Dedication waiver (http://creativeco mmons.org/publicdomain/zero/1.0/) applies to the data made available in this article, unless otherwise stated in a credit line to the data. 
Moreover, pancreatic resection is one of the most challenging surgery, with significant postoperative morbidity and mortality [10]. Improving preoperative status-by means of biliary drainage, hemostasis correction, prehabilitation with nutritional and adapted physical activity interventions [11] - is needed before surgery as complications are more frequent in unfit patients [12]. Shorter TTS may not allow to optimize prehabilitation but could improve carcinologic prognosis.

This study evaluated the impact of TTS on OS, DFS and postoperative complication rate in patients who underwent upfront curative intent surgical resection of a PA.

\section{Materials and methods}

We retrospectively included patients with upfront resected PA between January 1, 2004 and December 31, 2014 in three tertiary French centers (Reims University Hospital, Amiens University Hospital, Strasbourg University Hospital) and in one private center (Reims Courlancy Clinic). Patients were screened with administrative coding and multidisciplinary tumor board meetings data (MTBM). All cases have been discussed in MTBM, including a senior radiologist and a pancreatic surgeon, as now recommended by the consensus of the International Study Group of Pancreatic Surgery [13]. No systematic preoperative imaging review was made at inclusion.

All patients who underwent upfront curative intent resection for a PA were included. Patients were excluded in case of neoadjuvant treatment or incomplete surgical resection (R2).

Basic baseline clinical, biological (i.e. ECOG performance status (PS), initial symptoms, tumor location, preoperative biliary drainage, bilirubin levels and neutrophil-lymphocyte ratio), pathological and surgical data [i.e. TNM staging (5th, 6th or 7th edition, according to the standard of care at the time of treatment], lymph node ratio (number of invaded lymph nodes/total number of resected lymph nodes), resection margins [R0 or R1 $(<1 \mathrm{~mm})$ ], venous and adjacent organ involvement (gastric, colon or left adrenal resection), were collected using medical records. Follow up characteristics such as postoperative complications, adjuvant treatment regimen and evolution of the disease (tumor recurrence, site of recurrence and death) were also collected.

Time to decision (TTD) was defined as the delay in days between first specialized medical interview (gastroenterologist, pancreatic surgeon, or medical oncologist) and MTBM decision. Time to surgery (TTS) was defined as the delay between the first specialized medical interview (gastroenterologist, pancreatic surgeon, medical oncologist) and surgery. The 90-day postoperative complications were assessed using Dindo-Clavien classification and ISGPS definitions [14]. Grade III or higher grade complications were considered as severe [14]. Grade V complications correspond to postoperative death. Follow-up was standard and left to the physician's discretion according to guidelines prevailing at the time of the treatment [3]. The primary objective was to determine the impact of a shorter ( $\leq 14$ days) TTS in DFS improvement. This threshold was chosen in accordance with literature review and the investigators' experience [15-20]. Secondary objectives were to evaluate OS and DFS according to other TTS and TTD thresholds (7-, 14-, 28-, 60- and 75-day delay). Ninety-day morbidity rate was compared using a 14-day delay. Endpoint date was set to provide at least a 12-month follow-up (December 31, 2015).

\section{Statistical analysis}

Continuous variables were described as median and interquartile ranges. Categorical variables were described as frequencies expressed with percentages. Groups with different management delays were compared using Mann-Whitney test (non-normal continuous variables) and Chi square test or Fisher test (categorical variables), depending on variable type and sample size. OS was calculated from date of surgery procedure to date of death or censoring at the date of last visit. DFS was calculated from the date of the surgical procedure to the date of progression, death or censoring at the date of last visit [9]. Survival curves were established using the Kaplan-Meier method and compared using Cox proportional-hazards model for univariate and multivariate analyses. We preplanned several survival analyses stratified on different delay cut-offs (7, 14, 28, 60 and 75 days). Complication rates were analyzed according to a 14-day delay using Mann-Whitney test and logistic regression adjusted for factors with a $\mathrm{p}<0.2$ in stepwise regression. All data analyses were performed using $\mathrm{R}$ ( $\mathrm{R}$ Development Core Team, 2005). Statistical significance was defined as a $p$ value $<0.05$ for all tests.

\section{Ethics}

Patient's records were anonymized prior to analysis. Database was constituted in accordance with the reference methodology MR004 of the National Commission of Liberties and Informatics. (no. 2206749, 13/09/2018). As per French regulations, no additional ethical review was required.

\section{Results}

Population characteristics at baseline

A total of 534 patients with a PA were screened. Among them, 201 patients underwent an upfront surgery 
procedure with curative intent; 33 were finally excluded due to unresectable tumor upon surgical reassessment [21] or insufficient data collection [6] yielding a total of 168 included patients. The study flowchart is presented in Fig. 1 (Flowchart).

Patient characteristics are presented in Table 1. Most patients were men $(57.7 \%)$, with a median age of 66 years (IQR 58-71). ECOG PS was available in $60.2 \%$ of the cases. Only four patients were ECOG PS $>1$. Jaundice was the main symptom (69\%) at first patient visit, and diagnosis was made during a hospital stay in $44.6 \%$ of the cases. Tumors were mainly located in the head of the pancreas $(79.8 \%)$, warranting a biliary drainage in 64 patients (38.1\%). Nine of the patients underwent isolated metastases (liver and/or distant lymph nodes) resection, in curative intent at the time of surgery. Median total bilirubin prior to any biliary drainage was $197.5 \mu \mathrm{mol} / \mathrm{L}$ (IQR 135.5-336.75).

\section{Treatment characteristics}

Pancreaticoduodenectomy was performed in 131 patients, (78\%). Mesenteric superior vein was resected in 69 patients $(41.1 \%)$. Other organs were resected in only 10 patients (6\%) (Table 1B). Median length of hospital stay was 19 days (IQR 14-27.5). TNM stage on the final pathology report was mainly T3 (76.2\%) and N1 (76.8\%). A median of two lymph nodes were positive at pathological analysis (IQR 1-4) for a lymph node ratio calculated at 0.08 (IQR 0.00-0.2). Postoperative complication data were available in 159 patients (94.6\%). Eighty-seven $(51.8 \%)$ and 66 patients (39.3\%) experienced hemorrhage and fistulas, respectively. Post-operative complications were considered severe in 41 patients (24.4\%). Ten patients $(6 \%)$ died of post-operative complications (Dindo-Clavien class 5).

Adjuvant chemotherapy was administered in 141 patients (83.9\%), mainly gemcitabine. A median of 6(IQR 5.5-6) chemotherapy cycles were administered. was Adjuvant radiotherapy was used for 13 patients (7.7\%).

Median follow-up lasted 651.5 days (IQR 374, 1077.2), approximatively 21.5 months. Recurrence was identified for 128 patients $(76.2 \%)$, and classified as at least distant/ metastatic (95.3\%, 122 patients), or only locoregional (4.7\%, 6 patients). A total of 114 (67.9\%) patients had died at study endpoint date.

\section{Delay analysis}

Median TTD was 9.5 days (IQR 1-30.5). Median TTS was 19 days (IQR 12-36).

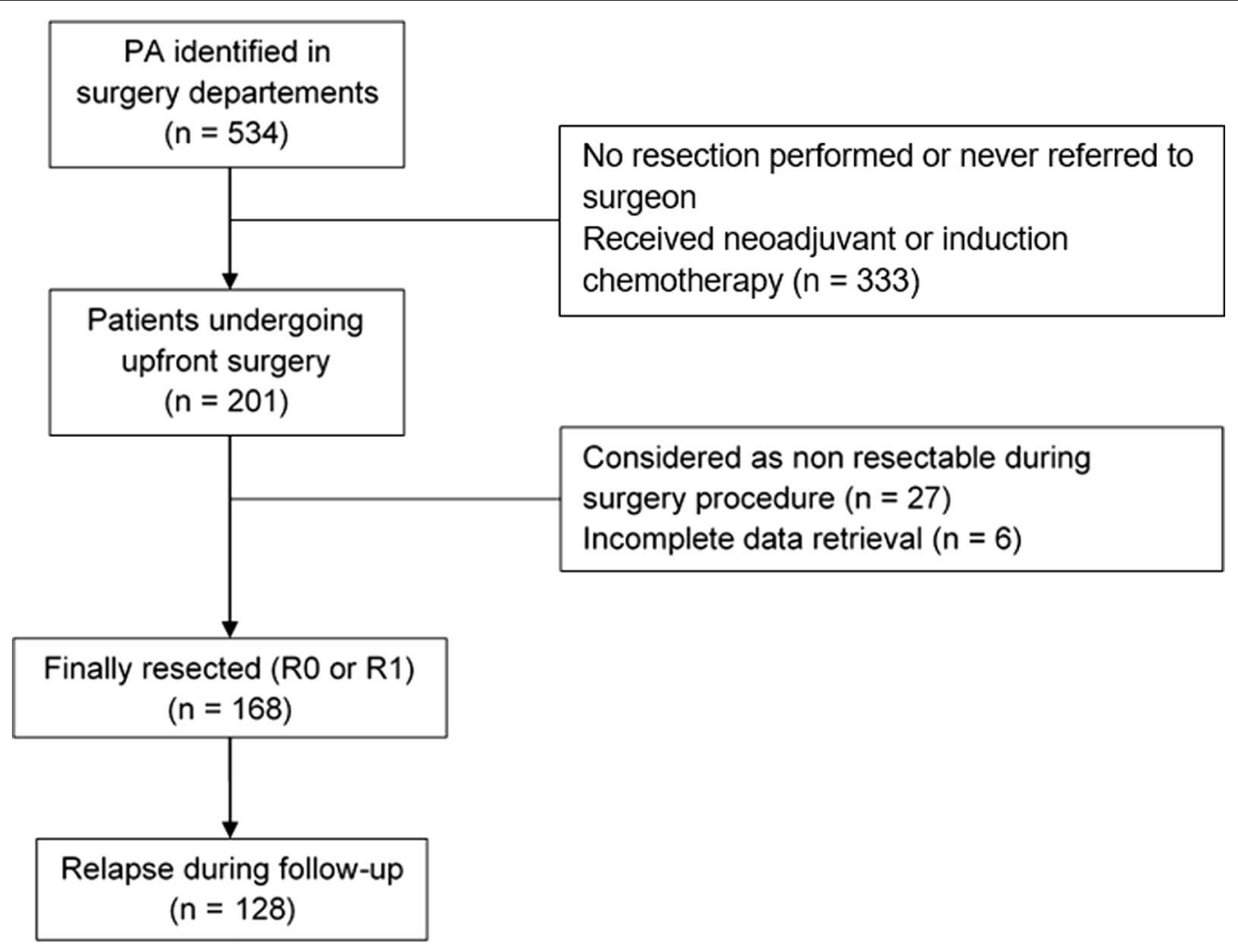

Fig. 1 Flowchart. $n$ total population; $R$ resection status 
Table 1 Study population characteristics

\begin{tabular}{|c|c|}
\hline \multicolumn{2}{|l|}{ A. Baseline characteristics } \\
\hline Characteristics & $n=168$ \\
\hline \multicolumn{2}{|l|}{ Inclusion center (\%) } \\
\hline Reims University Hospital & $70(41.7)$ \\
\hline Reims Courlancy Clinic & $12(7.1)$ \\
\hline Amiens University Hospital & $60(35.7)$ \\
\hline Strasbourg University Hospital & $26(15.5)$ \\
\hline Male $(\%)$ & $71(57.7)$ \\
\hline Age, years (median [IQR]) & $66[58.00,71.00]$ \\
\hline $\mathrm{BMI}, \mathrm{kg} / \mathrm{m}^{2}$ (median [IQR]) & $25.33[22.49,28.58]$ \\
\hline \multicolumn{2}{|l|}{ Performance status (\%) } \\
\hline 0 & $70(41.7)$ \\
\hline 1 & $27(16.1)$ \\
\hline 2 & $4(2.4)$ \\
\hline NA & $67(39.9)$ \\
\hline \multicolumn{2}{|l|}{ Clinical symptoms at presentation (\%) } \\
\hline Jaundice & $116(69.0)$ \\
\hline Abdominal pain & $53(31.5)$ \\
\hline Weight loss & $5(3.0)$ \\
\hline Incidentaloma & $6(3.6)$ \\
\hline Other & $26(15.5)$ \\
\hline NA & $1(0.6)$ \\
\hline Diagnosed during hospital stay (\%) & $75(44.6)$ \\
\hline \multicolumn{2}{|l|}{ Imaging technique used for diagnosis (\%) } \\
\hline Computed tomography & $85(50.6)$ \\
\hline Echography & $18(10.7)$ \\
\hline Echoendoscopy & $37(22.0)$ \\
\hline MRI & $21(12.5)$ \\
\hline Other & $5(3.0)$ \\
\hline NA & $2(1.2)$ \\
\hline \multicolumn{2}{|l|}{ Tumor location (\%) } \\
\hline Head & $134(79.8)$ \\
\hline Body & $13(7.7)$ \\
\hline Tail & $18(10.7)$ \\
\hline NA & $3(1.8)$ \\
\hline Lymphadenopathy at imaging (\%) & $27(16.1)$ \\
\hline Endoscopic biliary drainage (\%) & $58(34.5)$ \\
\hline Radiological biliary drainage (\%) & $6(3.6)$ \\
\hline Total bilirubin $(\mu \mathrm{mol} / \mathrm{L})($ median $[\mathrm{IQR}])$ & $54.00[9.47,185.25]$ \\
\hline Conjugated bilirubin ( $\mu \mathrm{mol} / \mathrm{L})$ (median [IQR]) & $46.00[7.20,148.50]$ \\
\hline Neutrophils to lymphocytes ratio (median [IQR] $)^{\dagger}$ & $2.67[2.00,3.82]$ \\
\hline Time to decision (days) (median [IQR]) & $9.50[1.00,30.50]$ \\
\hline Time to surgery (days) (median [IQR]) & $19.00[12.00,36.00]$ \\
\hline \multicolumn{2}{|c|}{ B. Surgery procedure, histopathological, and follow up characteristics } \\
\hline Characteristics & $n=168$ \\
\hline \multicolumn{2}{|l|}{ Type of resection (\%) } \\
\hline Pancreaticoduodenectomy & $131(78.0)$ \\
\hline Splenopancreatectomy & $28(16.7)$ \\
\hline Left pancreatectomy & $4(2.4)$ \\
\hline
\end{tabular}


Table 1 (continued)

\section{B. Surgery procedure, histopathological, and follow up characteristics}

\begin{tabular}{|c|c|}
\hline Characteristics & $n=168$ \\
\hline Total pancreatectomy & $5(3.0)$ \\
\hline Vein resection (\%) & $69(41.1)$ \\
\hline Organ resection (\%) $)^{\mathrm{a}, \mathrm{b}}$ & $10(6.0)$ \\
\hline \multicolumn{2}{|l|}{$\mathrm{T}(\%)$} \\
\hline 1 & $3(1.8)$ \\
\hline 2 & $25(14.9)$ \\
\hline 3 & $128(76.2)$ \\
\hline 4 & $9(5.4)$ \\
\hline$x$ & $1(0.6)$ \\
\hline NA & $2(1.2)$ \\
\hline \multicolumn{2}{|l|}{$N(\%)^{b}$} \\
\hline 0 & $37(22.0)$ \\
\hline 1 & $129(76.8)$ \\
\hline$x$ & $1(0.6)$ \\
\hline NA & $1(0.6)$ \\
\hline \multicolumn{2}{|l|}{$M(\%)^{a, b}$} \\
\hline 0 & $157(93.5)$ \\
\hline 1 & $9(5.4)$ \\
\hline$x$ & $2(1.2)$ \\
\hline Number of lymphatic nodes sampled (median [IQR]) & $19.00[12.00,25.00]$ \\
\hline Number of invaded lymph nodes (median [IQR] $)^{\mathrm{b}}$ & $2.00[1.00,4.00]$ \\
\hline Invaded/sampled lymph nodes (median [IQR]) & $0.08[0.00,0.20]$ \\
\hline \multicolumn{2}{|l|}{ Resection status $(\%)^{b}$} \\
\hline 0 & $105(62.5)$ \\
\hline 1 & $62(36.9)$ \\
\hline NA & $1(0.6)$ \\
\hline \multicolumn{2}{|l|}{ Microscopic vascular invasion (\%) } \\
\hline Yes & $103(61.3)$ \\
\hline No & $25(14.9)$ \\
\hline NA & $40(23.8)$ \\
\hline \multicolumn{2}{|l|}{ Perineural invasion (\%) } \\
\hline Yes & $137(81.5)$ \\
\hline No & $10(6.0)$ \\
\hline NA & $21(12.5)$ \\
\hline Length of stay (days) (median [IQR]) & $19.00[14.00,27.50]$ \\
\hline \multicolumn{2}{|l|}{ Postoperative complications (\%) } \\
\hline Yes & $109(64.9)$ \\
\hline No & $50(29.8)$ \\
\hline NA & $9(5.4)$ \\
\hline \multicolumn{2}{|l|}{ Hemorrhage (\%) $)^{a, b}$} \\
\hline Yes & $22(13.1)$ \\
\hline No & $87(51.8)$ \\
\hline NA & $59(35.1)$ \\
\hline \multicolumn{2}{|l|}{ Fistula (\%) $)^{a, b}$} \\
\hline Yes & $42(25.0)$ \\
\hline \multicolumn{2}{|l|}{ Fistula grade (\%) } \\
\hline $1 \mathrm{~A}$ & $22(52.4)$ \\
\hline $2 B$ & $13(31)$ \\
\hline
\end{tabular}


Table 1 (continued)

\begin{tabular}{|c|c|}
\hline \multicolumn{2}{|c|}{ B. Surgery procedure, histopathological, and follow up characteristics } \\
\hline Characteristics & $n=168$ \\
\hline $3 C$ & $6(14.3)$ \\
\hline NA & $1(2.4)$ \\
\hline No & $66(39.3)$ \\
\hline NA & $60(35.7)$ \\
\hline \multicolumn{2}{|l|}{ Dindo-Clavien classification (\%) } \\
\hline Benign & $124(73.8)$ \\
\hline 0 & $63(37.5)$ \\
\hline 1 & $18(10.7)$ \\
\hline 2 & $43(25.6)$ \\
\hline Severe ${ }^{a, b}$ & $41(24.4)$ \\
\hline $3 a$ & $11(6.5)$ \\
\hline $3 b$ & $13(7.7)$ \\
\hline $4 a$ & $5(3.0)$ \\
\hline $4 b$ & $2(1.2)$ \\
\hline 5 & $10(6.0)$ \\
\hline NA & $3(1.8)$ \\
\hline Follow-up (days) (median [IQR]) & $651.5[374,1077.2]$ \\
\hline Adjuvant chemotherapy (\%) & $141(83.9)$ \\
\hline Number of chemotherapy cycles (median [IQR]) & $6.00[5.50,6.00]$ \\
\hline Adjuvant radiotherapy (\%) & $13(7.7)$ \\
\hline Disease recurrence (\%) & $128(76.2)$ \\
\hline Local disease recurrence (\%) & $6(3.6)$ \\
\hline Lymph node recurrence (\%) & $37(40.7)$ \\
\hline Liver recurrence $(\%)^{\mathrm{a}}$ & $45(49.5)$ \\
\hline Peritoneal recurrence (\%) & $15(16.5)$ \\
\hline Other (\%) & $25(27.5)$ \\
\hline Disease-free survival (days) (median [IQR]) & $363.00[214.75,534.25]$ \\
\hline Death (\%) & $114(67.9)$ \\
\hline
\end{tabular}

$n$ total population; IQR interquartile range; $\mathrm{kg} / \mathrm{m}^{2}$ kilograms per square meter; $B M I$ body mass index; $N A$ not available; $M R I$ magnetic resonance imaging

${ }^{\text {a }}$ Variable associated with overall survival using univariate Cox proportional-hazards model

${ }^{b}$ Variable associated with disease free survival using univariate Cox proportional-hazards model

Patient characteristics stratified upon TTS inferior to 14 days are presented in Table 2 . A total of 59 patients (35\%) had their tumor resected less than 14 days after first consultation. Patients in the longer delay group had more frequent vein resections, endoscopic biliary drainage $(\mathrm{p}<0.001)$, jaundice $(\mathrm{p}=0.044)$, and higher median total serum bilirubin levels $(\mathrm{p}<0.001)$.

Univariate survival analysis using Cox model showed no significant statistical differences with a 14-day TTS threshold (Fig. 2) for DFS ( $\mathrm{p}=0.82)$ or OS $(\mathrm{p}=0.97)$. No difference was shown for DFS either with a $7-(\mathrm{p}=0.22)$, 28- $(\mathrm{p}=0.33), 60-(\mathrm{p}=0.79)$ or $75-(\mathrm{p}=0.88)$ day delay. OS and DFS were not different in both groups when comparing extreme delays (Table 3). TTD did not impact either DFS or OS, regardless of the delay cut-off (Table 3).
Adjusted OS (on organ and vein invasion, severe complications, and adjuvant chemotherapy) and adjusted DFS (on vein invasion, organ resection, severe complications, $\mathrm{R}$ status, $\mathrm{N}$ status, and adjuvant chemotherapy) were not significantly improved with a shorter TTS, inferior or equal to 14 days $(\mathrm{p}=0.44$ (Table 3 ).

Delay had no influence on the occurrence of fistula $(\mathrm{p}=0.44)$, hemorrhage $(\mathrm{p}=0.35)$ and severe postoperative complication $(\mathrm{p}=1)$ (Table 4). Multivariate logistic regression analysis adjusted on significant factors selected from the univariate analyses did not show any statistical significance concerning fistula $(\mathrm{p}=0.41)$, severe post-operative complication $(\mathrm{p}=0.82)$ or hemorrhage $(\mathrm{p}=0.59)($ Table 4$)$. 
Table 2 Study population characteristics stratified by a fourteen-day-time-to-surgery

\begin{tabular}{|c|c|c|c|}
\hline \multicolumn{4}{|l|}{ A. Baseline characteristics } \\
\hline Characteristics $(n=168)$ & $\leq 14$ days & $>14$ days & $p$ \\
\hline$n$ & 59 & 106 & \\
\hline Male (\%) & $38(64.4)$ & $58(54.7)$ & 0.334 \\
\hline Age (median [IQR]) & $65.00[57.00,69.50]$ & $67[59,73]$ & 0.109 \\
\hline BMI (median [IQR]) & $25.52[23.20,29.49]$ & $25.10[22.50,28.33]$ & 0.474 \\
\hline Performance status (\%) & & & 0.747 \\
\hline 0 & $23(65.7)$ & $47(71.2)$ & \\
\hline 1 & $10(28.6)$ & $17(25.8)$ & \\
\hline 2 & $2(5.7)$ & $2(3.0)$ & \\
\hline Jaundice (\%) & $47(79.7)$ & $67(63.2)$ & $0.044^{*}$ \\
\hline Diagnosed during hospital stay (\%) & $30(50.8)$ & $45(42.5)$ & 0.382 \\
\hline Tumor location (\%) & & & 0.3 \\
\hline Head & $51(87.9)$ & $82(78.1)$ & \\
\hline Body & $3(5.2)$ & $10(9.5)$ & \\
\hline Tail & $4(6.9)$ & $13(12.4)$ & \\
\hline Lymphadenopathy at imaging (\%) & $9(21.4)$ & $18(20.2)$ & 1 \\
\hline Endoscopic biliary drainage (\%) & $10(20.8)$ & $46(65.7)$ & $<0.001 *$ \\
\hline Radiological biliary drainage (\%) & $1(2.1)$ & $5(7.1)$ & 0.422 \\
\hline Total bilirubin ( $\mu \mathrm{mol} / \mathrm{L})($ median [IQR]) & $173.00[74.25,304.50]$ & $21.00[8.00,84.75]$ & $<0.001^{*}$ \\
\hline Conjugated bilirubin ( $\mu \mathrm{mol} / \mathrm{L})$ (median $[\mathrm{IQR}])$ & $140.50[60.50,236.25]$ & $22.00[4.00,76.50]$ & $<0.001^{*}$ \\
\hline Neutrophil-to-lymphocyte ratio (median [IQR]) & $2.67[2.06,4.09]$ & $2.68[1.92,3.52]$ & 0.223 \\
\hline
\end{tabular}

B. Surgery procedure, histopathological, and follow up characteristics stratified on a 14 days-time to surgery $n$ total population; IQR interquartile range; $N A$ not available; *statistical significance

\begin{tabular}{|c|c|c|c|}
\hline Characteristics $(n=168)$ & $\leq 14$ days & $>14$ days & $p$ \\
\hline$n$ & 59 & 106 & \\
\hline Type of resection (\%) & & & 0.497 \\
\hline Duodenopancreatectomy & $50(84.7)$ & $79(74.5)$ & \\
\hline Splenopancreatectomy & $7(11.9)$ & $20(18.9)$ & \\
\hline Left pancreatectomy & $1(1.7)$ & $3(2.8)$ & \\
\hline Total pancreatectomy & $1(1.7)$ & $4(3.8)$ & \\
\hline Vein resection (\%) & $16(27.1)$ & $52(49.1)$ & $0.01 *$ \\
\hline Organ resection (\%) & $4(6.8)$ & $6(5.7)$ & 1 \\
\hline $\mathrm{T}(\%)$ & & & 0.375 \\
\hline 1 & $0(0.0)$ & $3(2.9)$ & \\
\hline 2 & $6(10.2)$ & $18(17.3)$ & \\
\hline 3 & 49 (83.1) & $77(74.0)$ & \\
\hline 4 & $4(6.8)$ & $5(4.8)$ & \\
\hline$x$ & $0(0.0)$ & $1(1.0)$ & \\
\hline$N(\%)$ & & & 0.488 \\
\hline 0 & $11(18.6)$ & $26(24.8)$ & \\
\hline 1 & $48(81.4)$ & $78(74.3)$ & \\
\hline$x$ & $0(0.0)$ & $1(1.0)$ & \\
\hline M (\%) & & & 0.111 \\
\hline 0 & $53(89.8)$ & $101(96.2)$ & \\
\hline 1 & $6(10.2)$ & $3(2.9)$ & \\
\hline$x$ & $0(0.0)$ & $1(1.0)$ & \\
\hline Invaded/sampled lymph nodes (median [IQR]) & $0.06[0.00,0.15]$ & $0.10[0.00,0.20]$ & 0.403 \\
\hline Resection status $=1(\%)$ & $23(39.0)$ & $38(36.2)$ & 0.852 \\
\hline
\end{tabular}


Table 2 (continued)

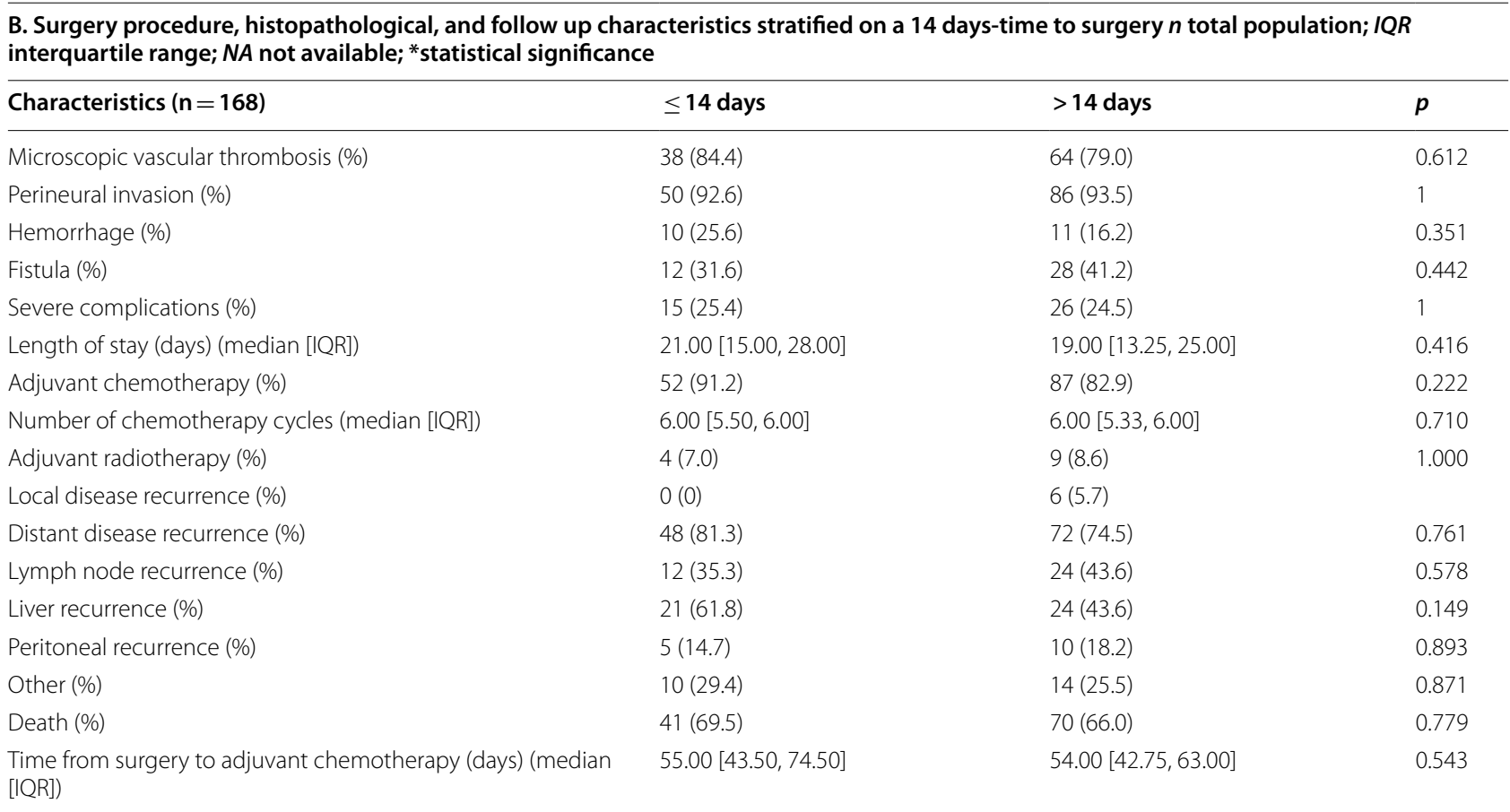

$n$ total population; IQR interquartile range; NA not available

*Statistical significance according to Mann-Whitney test (nonnormal continuous variables) or Chi-square test (categorical variable)

\section{Discussion}

In our study, TTS - considered as the time from the first specialized interview to surgery-had no impact on DFS and OS in patients with upfront resected PA. Hemorrhage, fistula, or severe post-operative complications rate were not different, regardless of TTS. This is the first study to evaluate the impact of TTS on the 90-day morbidity.

Shortening time to treatment has been a promising approach to improve survival. TTS has been set as a quality care index in other malignancies [6, 7, 22, 23]. As an example, HCC radiofrequency ablations warrant an under five-week management delay to avoid any impact on prognosis [6].

In PA, TTS impact remains unclear. Our results are consistent with most of the previous cohorts that showed no significant influence of TTS on survival rate for patients undergoing a curative intent resection for PA $[15,17,24]$. Eshuis et al. concluded that biliary drainage and prolonged TTS do not impair survival rates in a randomized controlled trial [25]. On the contrary, Swords et al. showed a modestly improved OS (1.8 months) and a higher 30 and 90-day post-operative mortality in shorter TTS, but at the cost of including a large number of patients [26]. Subgroup analysis suggested that shorter TTS could improve resecability rate and prognosis in small tumors [15, 26, 27]. Finally, none of these studies analyzed the consequences of shorter TTS on postoperative complications such as hemorrhage, fistulas or DindoClavien classification.

Published studies, including ours, have failed to demonstrate a significant survival benefit with shorter delays before upfront surgery in PA. However, PA arises from pancreatic parenchyma decades before being symptomatic and diagnosed, suggesting slow growth at early stages [21]. There is also evidence to support the rapid growth of PA, in later natural history once diagnosed, with an estimated time to progression from a $\mathrm{T} 1$ to a $\mathrm{T} 4$ stage of approximately 14 months [28]. Tumor volume growth could thereby be considered as a factor distinct from the disease stage. Marchegiani et al. showed a TTS effect on survival for smaller tumors (T1 and T2) [27]. Shorter TTS could impact resecability and disease-free survival in this specific patient subgroup. In our study, only 28 patients $(16.7 \%)$ had a T1 or T2 disease stage, making subgroup analyses futile.

Nevertheless, median TTS seems to be increasing year after year due to the incremental PA incidence and the frequent referral to expert centers [22]. As TTS has no or little impact on prognosis, management strategy could be modified to allow sufficient time to confirm diagnosis in ambivalent cases and integrate preoperative 
2A. Disease-free survival according to time to surgery $(p=0.82)$;

+ Over 14 days + Below 14 days

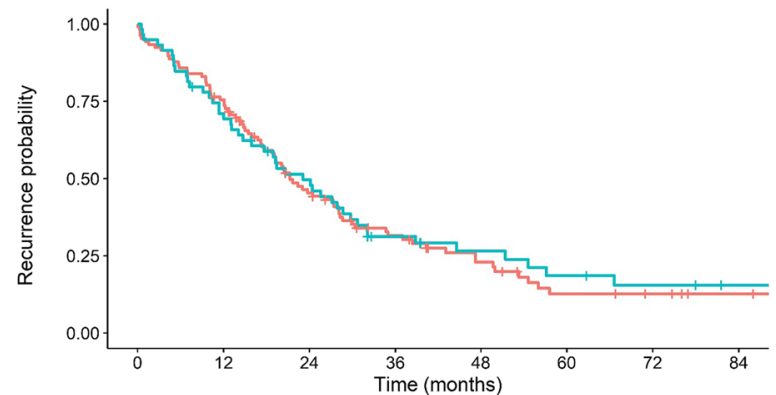

Number at risk

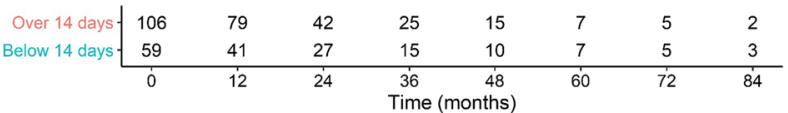

$2 C$. Disease free survival according to time to decision $(p=0.56)$;

+ Over 14 days + Below 14 days

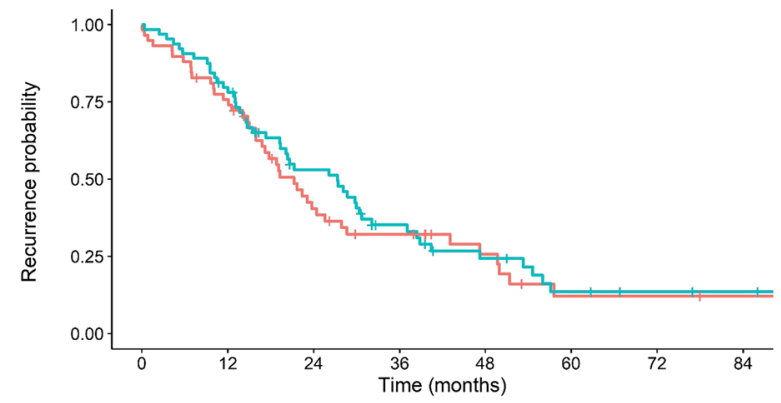

Number at risk

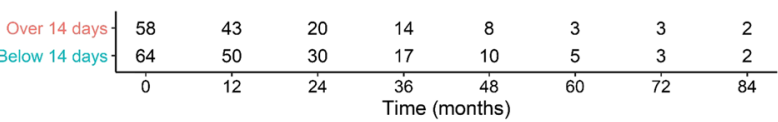

$2 B$. Overall survival according to time to surgery $(p=0.97)$;

+ Over 14 days + Below 14 days

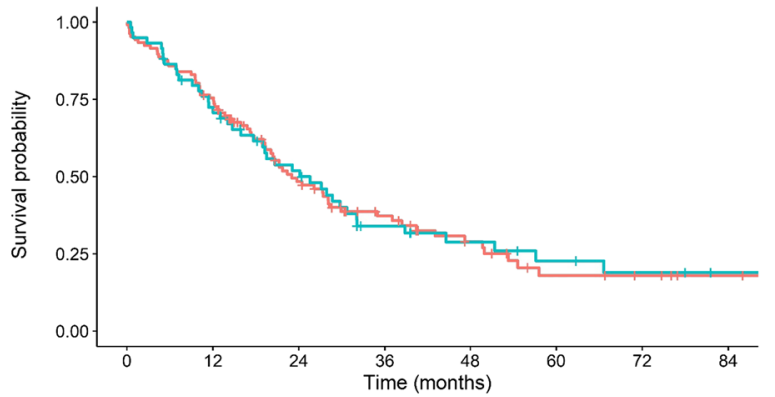

Number at risk

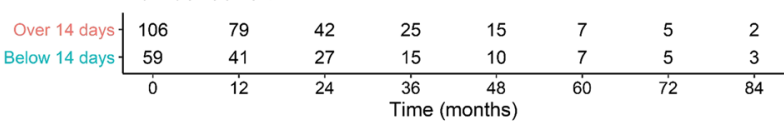

2D. Overall survival according to time to decision $(p=0.5)$;

+ Over 14 days + Below 14 days

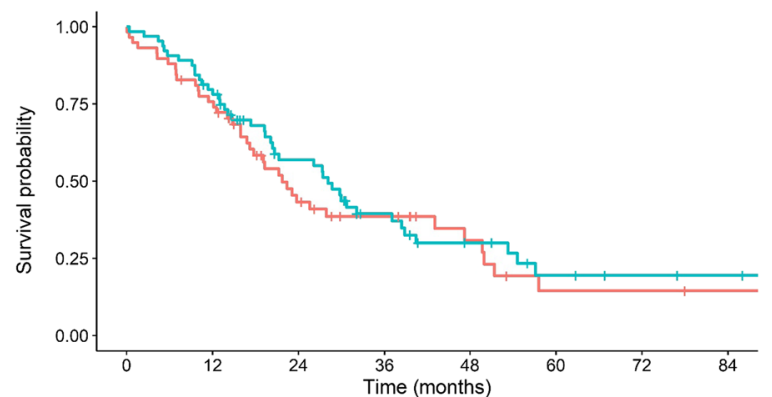

Number at risk

\begin{tabular}{|c|c|c|c|c|c|c|c|c|}
\hline Over 14 days & 58 & 43 & 20 & 14 & 8 & 3 & 3 & 2 \\
\hline Below 14 days. & 64 & 50 & 30 & 17 & 10 & 5 & 3 & 2 \\
\hline & 0 & 12 & 24 & 36 & 48 & 60 & 72 & 84 \\
\hline
\end{tabular}

Fig. 2 Disease-free and overall survival according to time to surgery and time to decision. A Disease-free survival according to time to surgery $(p=0.82) ; \mathbf{B}$ Overall survival according to time to surgery $(p=0.97) ; \mathbf{C}$ Disease free survival according to time to decision $(p=0.56) ; \mathbf{D}$ Overall survival according to time to decision $(p=0.5)$

chemotherapy and prehabilitation strategy. A 28-day minimum delay between last liver imaging and surgery remains well established to avoid any curative surgery performed on patients with liver metastases [3].

No link was observed for fistula, hemorrhage, or severe post-operative complications when TTS was shortened. Post pancreatectomy hemorrhage is mainly due to anastomotic leaks causing pseudoaneurysms [29]. Also, when performed early, these interventions are less likely to be the subject of technical debate in multidisciplinary surgical meetings and patients may be less well prepared (uncorrected coagulation, nutritional status, or jaundice). Moreover, significantly higher median bilirubin in the shorter delay group can lead to troubled hemostasis, facilitating post-operative hemorrhage [30].

Our study has several limitations. First, patients with macroscopically incomplete resection (R2) or those who finally did not undergo a curative surgery, despite an initially resectable primary tumor, were not included. These patients could potentially have longer delays explaining tumor progression. Unfortunately, they were not considered in our analysis avoiding performing an intention-totreat analysis. Vein resection rate was higher in the over 14-day delay group. This significative difference could be explained by a higher number of patients with borderline resectable disease in this group. Unfortunately, no systematic preoperative imaging review was performed, which did not allow us to better assess those patients. Furthermore, patients who waited longer without any treatment but finally underwent a curative surgical procedure may have had slower-progressive disease with a better prognosis. Preselecting candidates for curative surgery on their time to progression in the setting of such an aggressive disease seems unethical. Also, 116 patients 
Table 3 Overall and disease-free survival analyses according to different times to decision and time-to-surgery cut-offs

\begin{tabular}{|c|c|c|c|c|c|c|c|c|}
\hline \multirow[t]{2}{*}{ Cut-off } & \multicolumn{2}{|c|}{ Number of patients } & \multicolumn{3}{|c|}{ Overall survival } & \multicolumn{3}{|c|}{ Disease-free survival } \\
\hline & Lower cut-off & Higher cut-off & $\mathbf{p}$ & OR & $\mathrm{Cl} 95 \%$ & $\mathbf{p}$ & OR & $\mathrm{Cl} 95 \%$ \\
\hline \multicolumn{9}{|l|}{ Univariate analysis } \\
\hline \multicolumn{9}{|l|}{ Time to decision } \\
\hline$\leq 7$ days vs. $>7$ days & 18 & 147 & 0.59 & 0.89 & $0.57-1.38$ & 0.56 & 0.88 & $0.58-1.34$ \\
\hline$\leq 14$ days vs. $>14$ days & 59 & 106 & 0.50 & 0.86 & $0.55-1.34$ & 0.56 & 0.88 & $0.58-1.34$ \\
\hline$\leq 28$ days vs. $>28$ days & 104 & 61 & 0.79 & 1.07 & $0.65-1.76$ & 0.68 & 1.10 & $0.69-1.8$ \\
\hline$\leq 60$ days vs. $>60$ days & 151 & 14 & 0.78 & 0.88 & $0.35-2.19$ & 0.79 & 1.09 & $0.6-1.97$ \\
\hline$\leq 7$ days vs. $>28$ days & 18 & 61 & 0.96 & 0.98 & $0.58-1.68$ & 0.97 & 1.01 & $0.61-1.68$ \\
\hline \multicolumn{9}{|l|}{ Time to surgery } \\
\hline$\leq 7$ days vs. $>7$ days & 18 & 147 & 0.21 & 1.43 & $0.82-2.52$ & 0.22 & 1.4 & $0.81-2.41$ \\
\hline$\leq 14$ days vs. $>14$ days & 59 & 106 & 0.97 & 0.99 & $0.67-1.46$ & 0.82 & 0.95 & $0.66-1.39$ \\
\hline$\leq 28$ days vs. $>28$ days & 104 & 61 & 0.38 & 1.2 & $0.80-1.77$ & 0.33 & 1.21 & $0.83-1.76$ \\
\hline$\leq 60$ days vs. $>60$ days & 151 & 14 & 0.88 & 0.95 & $0.52-1.74$ & 0.79 & 1.09 & $0.6-1.97$ \\
\hline$\leq 75$ days vs. $>75$ days & 157 & 8 & 0.84 & 0.93 & $0.3-1.99$ & 0.88 & 1.06 & $0.49-2.28$ \\
\hline$\leq 7$ days vs. $>60$ days & 18 & 14 & 0.34 & 1.5 & $0.66-3.41$ & 0.26 & 1.6 & $0.71-3.59$ \\
\hline \multicolumn{9}{|l|}{ Multivariate analysis $^{\mathrm{a}}$} \\
\hline \multicolumn{9}{|l|}{ Time to surgery } \\
\hline$\leq 14$ days vs. $>14$ days & 59 & 104 & 0.44 & 1.18 & $0.76-1.82$ & 0.99 & 1.00 & $0.66-1.51$ \\
\hline
\end{tabular}

vs versus; OR odds ratio; Cl 95\% confidence interval 95\%

${ }^{a}$ Adjusted analysis for vein resection, jaundice, and hemorrhage

Table 4 Univariate and multivariate analysis comparing the 90-day postoperative morbidity for a 14-days time to surgery

\begin{tabular}{|c|c|c|c|c|c|}
\hline & \multirow{2}{*}{$\begin{array}{l}\leq 14 \text { days }(N=59) \\
n(\%)\end{array}$} & \multirow{2}{*}{$\begin{array}{l}>14 \text { days }(\mathrm{N}=106) \\
\mathrm{n}(\%)\end{array}$} & \multirow{2}{*}{$\begin{array}{l}\text { Univariate } \\
\text { analysis } \\
\mathbf{p}\end{array}$} & \multicolumn{2}{|c|}{ Multivariate analysis } \\
\hline & & & & $\mathbf{p}$ & OR [Cl95\%] \\
\hline Hemorrhage (versus absence) ${ }^{\mathrm{a}}$ & $10(25.6)$ & $11(16.2)$ & 0.351 & 0.59 & $1.34[0.46-3.94]$ \\
\hline Fistula (versus absence) $)^{b}$ & $12(31.6)$ & $28(41.2)$ & 0.442 & 0.41 & $0.66[0.24-1.16]$ \\
\hline Severe complications (versus Clavien $<3)^{c}$ & $15(25.4)$ & $26(24.5)$ & 1 & 0.82 & $0.75[0.06-9]$ \\
\hline
\end{tabular}

$n$ number of patient; $N$ total per category

${ }^{\text {a }}$ Adjusted for jaundice, superior mesenteric vein invasion, and T status

${ }^{\mathrm{b}}$ Adjusted for jaundice, tumor localization and type of resection

${ }^{c}$ Adjusted for body mass index, lymph node invasion, invaded/sampled lymph nodes ratio and superior mesenteric vein invasion

(69\%) were diagnosed upon jaundice presentation, while only $64(38.1 \%)$ experienced a radiological or endoscopic biliary drainage. Patients treated before 2010 were more frequently resected with higher bilirubin levels. Moreover, the power of the study could be insufficient due to the limited number of inclusion centers and retrospectively included patients. Finally, three of the four inclusion centers, including $92.9 \%$ of the patients, were tertiary hospitals. We cannot exclude a selection bias and a confusion effect due to highly skilled techniques developed in these centers with low complication rates.

Surgery remains the only existing treatment to cure PA [3]. Recent progress with polychemotherapy regimens and better patient selection for surgery has modestly improved overall survival [31, 32]. Moreover, new management strategies including neoadjuvant and induction chemotherapies are to be interpreted differently from passive delay where no therapeutic interventions occur. New perspectives must be found to increase the survival and quality of life of these patients. PA may not be the most suitable malignancy to study TTS as a quality metric. However, the COVID-19 pandemic has called for urgent case hierarchization and has thereby raised new questions about carcinologic surgical priorities. Ongoing multicentric CAPANCOVID-19 (https://clinicaltr ials.gov/ct2/show/NCT04406571) tries to measure the impact of the COVID-19 pandemic causing prolonged management delay, from surgery to palliative situations. 
We showed that TTS seems to have no impact on OS, DFS and 90-day postoperative morbidity in patients with resected pancreatic adenocarcinoma. Other trials need to be carried out to understand the role of TTS in smaller tumor sizes.

\section{Acknowledgements}

Université de Reims Champagne Ardennes supported this study. The authors thank the technical assistance of Grace Stockton-Bliard for English editing.

\section{Authors' contributions}

MB performed all statistical analyzes including tables and figures, wrote, reviewed and edited the original and final draft. OB, RK and SD conceptualized this study, supervised, validated, wrote, reviewed, and edited the final draft. LT conceptualized the study, collected data, reviewed, and edited the final draft. AT wrote, reviewed and edited the original and final draft. JR, PP, BR, RR, CP CN and TP supervised, reviewed and validated the final draft. All authors read and approved the final manuscript.

\section{Funding}

No funding was obtained for this study.

\section{Availability of data and materials}

The datasets used and/or analysed during the current study are available from the corresponding author on reasonable request.

\section{Declarations}

\section{Ethics approval and consent to participate}

The institutional review board at Reims University Hospital approved this study. Patients' verbal consents were collected in accordance with the protocol MR004 from the Commission Nationale de I'Informatique et des Libertés (no. 2206749, 13/09/2018), and followed the French authorities' requirements.

\section{Consent for publication}

Not applicable.

\section{Competing interests}

M Brugel, R Kianmanesh, L Teuma, A Tashkandi, B Royer, R Rhaiem, C Perrenot, T Piardi, S Deguelte: none; O Bouché: speaker honoraria/advisory role for Merck KGaA, Roche Genentech, Bayer, Astra-Zeneca, Grunenthal, MSD, Amgen, Pierre Fabre, Servier outside the submitted work, JM Regimbeau: advisory role for Quantum Robotics outside the submitted work; P Pessaux: orator for Integra and 3M, co-founder of Virtualisurg outside the submitted work; C Neuzillet: consultancy/honoraria: Pierre Fabre, Servier, Roche, AstraZeneca, Bristol-Myers Squibb, Amgen, Merck, MSD, Novartis, Incyte Biosciences, Mylan, Baxter, Nutricia, Fresenius Kabi; research funding: Roche; clinical trials: OSE Immunotherapeutics, AstraZeneca, Bristol-Myers Squibb outside the submitted work.

\section{Author details}

${ }^{1}$ Department of Ambulatory Oncology Care Unit, Centre Hospitalier Universitaire de Reims, Rue du general Koenig, Reims, France. ${ }^{2}$ University Reims Champagne-Ardenne (URCA), Reims, France. ${ }^{3}$ Digestive and Endocrine Surgery Department, Centre Hospitalier Universitaire de Reims, Rue du général Koenig, Reims, France. ${ }^{4}$ Digestive Surgery Department, CHU Amiens Picardie, 1 rond-point du Professeur Christian Cabrol, Amiens, France. ${ }^{5}$ University of Picardie Jules-Vernes, 51 boulevard de Chateaudun, Amiens, France. ${ }^{6}$ General, Digestive, and Endocrine Surgery, Nouvel Hôpital Civil, 1 quai Louis Pasteur, Strasbourg, France. ${ }^{7}$ Université de Strasbourg, Strasbourg, France. ${ }^{8}$ General Surgeon, Clinique de Courlancy, 38bis rue de Courlancy, Bezannes, France. ${ }^{9}$ Medical Oncology Department, Institut Curie, 35 rue Dailly, Saint-Cloud, France. ${ }^{10}$ Versailles Saint-Quentin University, Paris Saclay University, Saint-Cloud, France.

Received: 1 July 2021 Accepted: 16 November 2021 Published online: 07 December 2021

\section{References}

1. Rawla P, Sunkara T, Gaduputi V. Epidemiology of pancreatic cancer: global trends, etiology and risk factors. World J Oncol. 2019;10(1):10-27.

2. Rahib L, Smith BD, Aizenberg R, Rosenzweig AB, Fleshman JM, Matrisian LM. Projecting cancer incidence and deaths to 2030: the unexpected burden of thyroid, liver, and pancreas cancers in the United States. Cancer Res. 2014;74(11):2913-21.

3. Neuzillet C, Gaujoux S, Williet N, Bachet J-B, Bauguion L, Colson Durand L, et al. Pancreatic cancer: French clinical practice guidelines for diagnosis, treatment and follow-up (SNFGE, FFCD, GERCOR, UNICANCER, SFCD, SFED, SFRO, ACHBT, AFC). Dig Liver Dis déc. 2018;50(12):1257-71.

4. Conroy T, Desseigne F, Ychou M, Bouche O, Guimbaud R, Bécouarn Y, et al. FOLFIRINOX versus gemcitabine for metastatic pancreatic cancer. N Engl J Med. 2011;9:1817.

5. Santos F, Dragomir A, Kassouf W, Franco E, Aprikian A. Urologist referral delay and its impact on survival after radical cystectomy for bladder cancer. Curr Oncol Tor Ont févr. 2015;22(1):e20-26.

6. Chen W-T, Fernandes ML, Lin C-C, Lin S-M. Delay in treatment of earlystage hepatocellular carcinoma using radiofrequency ablation may impact survival of cirrhotic patients in a surveillance program. J Surg Oncol. 2011;103(2):133-9.

7. Eaglehouse YL, Georg MW, Shriver CD, Zhu K. Time-to-surgery and overall survival after breast cancer diagnosis in a universal health system. Breast Cancer Res Treat. 2019;178(2):441-50.

8. Hanna TP, King WD, Thibodeau S, Jalink M, Paulin GA, Harvey-Jones E, et al. Mortality due to cancer treatment delay: systematic review and meta-analysis. BMJ. 2020;371:4087.

9. Lukács G, Kovács Á, Csanádi M, Moizs M, Repa I, Kaló Z, et al. Benefits of timely care in pancreatic cancer: a systematic review to navigate through the contradictory evidence. Cancer Manag Res. 2019;11:9849-61.

10. Swanson RS, Pezzi CM, Mallin K, Loomis AM, Winchester DP. The 90-day mortality after pancreatectomy for cancer is double the 30-day mortality: more than 20,000 resections from the National Cancer Data Base. Ann Surg Oncol. 2014;21(13):4059-67.

11. Neuzillet C, Anota A, Foucaut A, Védie A, Antoun S, Barnoud D, et al. Nutrition and physical activity: French Intergroup Clinical Practice Guidelines for diagnosis, treatments and follow-up (SNFGE, FFCD, GERCOR, UNICANCER, SFCD, SFED, SFRO, ACHBT, AFC, SFP-APA, SFNCM, AFSOS). BMJ Support Palliat Care. 2020;11:381-95.

12. Bates A, West MA, Jack S. Framework for prehabilitation services: framework for prehabilitation services. Br J Surg. 2020;107(2):e11-4.

13. Bockhorn M, Uzunoglu FG, Adham M, Imrie C, Milicevic M, Sandberg AA, et al. Borderline resectable pancreatic cancer: a consensus statement by the International Study Group of Pancreatic Surgery (ISGPS). Surgery. 2014;155(6):977-88.

14. Dindo D, Demartines N, Clavien P-A. Classification of surgical complications. Ann Surg. 2004;240(2):205-13.

15. Sanjeevi S, Ivanics T, Lundell L, Kartalis N, Andrén-Sandberg $\AA$, Blomberg J, et al. Impact of delay between imaging and treatment in patients with potentially curable pancreatic cancer. Br J Surg. 2016;103(3):267-75.

16. Yun YH, Kim YA, Min YH, Park S, Won YJ, Kim DY, et al. The influence of hospital volume and surgical treatment delay on long-term survival after cancer surgery. Ann Oncol. 2012;23(10):2731-7.

17. Raptis DA, Fessas C, Belasyse-Smith P, Kurzawinski TR. Clinical presentation and waiting time targets do not affect prognosis in patients with pancreatic cancer. Surg J R Coll Surg. 2010;8(5):239-46.

18. GERCOR - Multidisciplinary Oncology Cooperative Group. Management of Symptomatic Advanced Pancreatic Adenocarcinoma: « Urgence Pancreas »-A Prospective A.R.CA.D Cohort Study [Internet]. clinicaltrials. gov; 2019 févr [cité 7 oct 2020]. Report No.: NCT02979483. Disponible sur: https://clinicaltrials.gov/ct2/show/NCT02979483

19. Neal RD, Tharmanathan P, France B, Din NU, Cotton S, Fallon-Ferguson $J$, et al. Is increased time to diagnosis and treatment in symptomatic cancer associated with poorer outcomes? Systematic review. Br J Cancer. 2015;112(Suppl 1):S92-107.

20. Kaltenmeier C, Shen C, Medich DS, Geller DA, Bartlett DL, Tsung A, et al. Time to Surgery and Colon Cancer Survival in the United States. Ann Surg [Internet]. 18 sept 2020 [cité 21 sept 2020];Publish Ahead of Print. Disponible sur: https://journals.Iww.com/annalsofsurgery/Abstract/9000/ Time_to_Surgery_and_Colon_Cancer_Survival_in_the.94742.aspx 
21. Wente MN, Veit JA, Bassi C, Dervenis C, Fingerhut A, Gouma DJ, et al. Postpancreatectomy hemorrhage (PPH)-An International Study Group of Pancreatic Surgery (ISGPS) definition. Surgery. 2007;142(1):20-5.

22. Paterson WG, Depew WT, Paré P, Petrunia D, Switzer C, van Zanten SJV, et al. Canadian consensus on medically acceptable wait times for digestive health care. Can J Gastroenterol. 2006;20(6):411-23.

23. Jooste V, Dejardin O, Bouvier V, Arveux P, Maynadie M, Launoy G, et al. Pancreatic cancer: wait times from presentation to treatment and survival in a population-based study. Int J Cancer. 2016;139(5):1073-80.

24. Swords DS, Zhang C, Presson AP, Firpo MA, Mulvihill SJ, Scaife CL. Association of time-to-surgery with outcomes in clinical stage I-II pancreatic adenocarcinoma treated with upfront surgery. Surgery. 2018;163(4):753-60

25. Marchegiani G, Andrianello S, Perri G, Secchettin E, Maggino L, Malleo $G$, et al. Does the surgical waiting list affect pathological and survival outcome in resectable pancreatic ductal adenocarcinoma? HPB. 2018;20(5):411-7.

26. Yachida S, Jones S, Bozic I, Antal T, Leary R, Fu B, et al. Distant metastasis occurs late during the genetic evolution of pancreatic cancer. Nature. 2010;467(7319):1114-7.

27. Yu J, Blackford AL, dal Molin M, Wolfgang CL, Goggins M. Time to progression of pancreatic ductal adenocarcinoma from low-to-high tumour stages. Gut. 2015;64(11):1783-9.

28. Papadopoulos V, Filippou D, Manolis E, Mimidis K. Haemostasis impairment in patients with obstructive jaundice. J Gastrointest Liver Dis JGLD. 2007;16(2):177-86.

29. Mattevi C, Garnier J, Marchese U, Ewald J, Gilabert M, Poizat F, et al. Has the non-resection rate decreased during the last two decades among patients undergoing surgical exploration for pancreatic adenocarcinoma? BMC Surg. 2020;20(1):176.

30. Conroy T, Hammel P, Hebbar M, BenAbdelghani M, Wei AC, Raoul J-L, et al. FOLFIRINOX or gemcitabine as adjuvant therapy for pancreatic cancer. N Engl J Med. 2018;379(25):2395-406.

31. Mirkin KA, Hollenbeak CS, Wong J. Time to surgery: a misguided quality metric in early stage pancreatic cancer. J Gastrointest Surg. 2018;22(8):1365-75

32. Eshuis WJ, van der Gaag NA, Rauws EAJ, van Eijck CHJ, Bruno MJ, Kuipers EJ, et al. Therapeutic delay and survival after surgery for cancer of the pancreatic head with or without preoperative biliary drainage. Ann Surg. 2010;252(5):840-9.

\section{Publisher's Note}

Springer Nature remains neutral with regard to jurisdictional claims in published maps and institutional affiliations.

Ready to submit your research? Choose BMC and benefit from:

- fast, convenient online submission

- thorough peer review by experienced researchers in your field

- rapid publication on acceptance

- support for research data, including large and complex data types

- gold Open Access which fosters wider collaboration and increased citations

- maximum visibility for your research: over 100M website views per year

At BMC, research is always in progress.

Learn more biomedcentral.com/submissions 